COMPARACIÓN DE 3 ECUACIONES PARA ESTIMAR EL GASTO ENERGÉTICO EN REPOSO VS CALORIMETRÍA INDIRECTA EN ESCOLARES CON OBESIDAD.

\author{
COMPARISON OF 3 EQUATIONS TO ESTIMATE THE ENERGY EXPENDITURE AT RESTING VS INDIRECT \\ CALORIMETRY IN SCHOOLCHILDREN WITH OBESITY.
}

Ramírez Resendez Beatriz ${ }^{1}$, Balderas Dibildox Daniela ${ }^{2}$, Sánchez Peña María Alejandra ${ }^{1}$, Márquez Zamora Leticia ${ }^{1}$, Ramírez López Erik ${ }^{1}$, López-Cabanillas Lomelí Manuel ${ }^{1}$, Solís Pérez Elizabeth ${ }^{1}$.

1 Facultad de Salud Pública y Nutrición, Universidad Autónoma de Nuevo León. 2 Consultoría Privada. Monterrey, N.L. México.

Citation: Ramírez Resendez B., Balderas Dibildox D., Sánchez Peña MA., Márquez Zamora L., Ramírez López E., López-Cabanillas Lomelí M., Solís Pérez E. (2020) Comparación de 3 Ecuaciones para estimar el Gasto Energético en Reposo Vs Calorimetría Indirecta en Escolares con Obesidad. Revista Salud Pública y Nutrición, 19 (2), 1-8.

Editor: Esteban G. Ramos Peña, Dr. CS., Universidad Autónoma de Nuevo León, Facultad de Salud Pública y Nutrición, Monterrey Nuevo León, México. Copyright: (C)2020 Ramírez Resendez B. et al. This is an open-access article distributed under the terms of Creative Commons Attribution License [CC BY 4.0], which permits unrestricted use, distribution, and reproduction in any medium, provided the original author and source are credited.

Competing interests: The authors have declared that no competing interests exist.

DOI: https://doi.org/10.29105/respyn19.2-1

Recibido: 09 de marzo 2020;

Aceptado: 12 de junio 2020

Email: maria.sanchezp@uanl.mx. 


\title{
COMPARACIÓN DE 3 ECUACIONES PARA ESTIMAR EL GASTO ENERGÉTICO EN REPOSO VS CALORIMETRÍA INDIRECTA EN ESCOLARES CON OBESIDAD.
}

\author{
Beatriz Ramírez Resendez ${ }^{1}$, Daniela Balderas Dibildox ${ }^{2}$, María Alejandra Sánchez Peña ${ }^{1}$, Leticia Márquez \\ Zamora ${ }^{1}$, Erik Ramírez López ${ }^{1}$, Manuel López-Cabanillas Lomeli ${ }^{1}$, Elizabeth Solís Pérez ${ }^{1}$.
}

1 Facultad de Salud Pública y Nutrición, Universidad Autónoma de Nuevo León. 2 Consultoría Privada. Monterrey, N.L. México.

\begin{abstract}
RESUMEN
Introducción. El gasto energético en reposo (GER) se determina por calorimetría indirecta; o por ecuaciones de predicción. Objetivo: Estimar el gasto energético en reposo por ecuaciones de predicción comparada con calorimetría indirecta en escolares con obesidad. Material y Método: Estudio comparativo, descriptivo y trasversal bajo previo consentimiento informado a escolares entre 7 y 12 años de edad con obesidad (> 2 D. E. OMS 2007). Bajo previo ayuno y reposo de 12 horas se realizó el estudio de calorimetría indirecta con el equipo Quark $\mathrm{RMR}^{\circledR}$. Las ecuaciones de predicción para estimar gasto energético fueron Schofield, Tverskaya y Lazzer. Se realizó el análisis estadístico con el software MedCalc ${ }^{\circledR}$ V13.3. Resultados: 41 escolares, $59 \%(n=24)$ género femenino y $41 \%(n=17)$ masculino, con promedio de edad de $9.0 \pm 2.0$ años. Al comparar el gasto energético, el promedio de las diferencias fue de $232 \mathrm{kcal}, 89 \mathrm{kcal}$ y $251 \mathrm{kcal}$ respectivamente; siendo la equivalencia entre grupos significativa para la ecuación predictiva de Schofield $(p=<0.0001)$, Tverskaya $(p=<0.0028)$ y Lazzer $(p=<0.0001)$. Conclusiones: Para determinar el GER en la población infantil con obesidad la mejor herramienta es calorimetría indirecta, ya que en termino grupal las tres ecuaciones de predicción sobreestiman significativamente el gasto energético en reposo. Palabras Clave: Calorimetría indirecta, Ecuaciones predictivas, Pediatría, Obesidad, Gasto energético en reposo.
\end{abstract}

\section{ABSTRACT}

Introduction: The energy expenditure at rest (GER) is a determined by indirect calorimetry, or by prediction equations. Objective: To estimate the energy expenditure at resting by prediction equations compared with indirect calorimetry in schoolchildren with obesity. Material and method: Comparative, descriptive and cross sectional study with prior informed consent to schoolchildren between 7 and 12 years of age with obesity (>2 D.E. WHO 2007). Under previous fasting and resting for 12 hours, an indirect calorimetry study was performed with the Quark RMR ${ }^{\circledR}$ equipment. The prediction equations to estimate energy expenditure were Schofield, Tverskaya and Lazzer. Statistical analysis was performed with MedCalc ${ }^{\circledR}$ V13.3 software. Results: 41 schoolchildren, $59 \%(n=24)$ female gender and $41 \%(n=17)$ male with an average age of $9.0+2.0$ years. When comparing energy expenditure, the average of the differences was $232 \mathrm{kcal}, 89 \mathrm{kcal}$ and $252 \mathrm{kcal}$ respectively; the equivalence between groups being significant for the predictive equation of Schofield $(p=<0.0001)$, Tverskaya $(p=<0.0028)$ and Lazzer $(p=<0.0001)$. Conclusions: To determine the GER in the obese child population, the best tool is indirect calorimetry, since in group terms the three prediction equations significantly overestimate the energy expenditure.

Key words: Indirect calorimetry, Predictive equation, Pediatrics, Obesity, Energy expenditure at resting. 


\section{Introducción}

El gasto energético en reposo (GER) se define como la energía necesaria para mantener las funciones fisiológicas esenciales (Stubelj et al., 2020).

Su desequilibrio entre la ingesta y la utilización de la misma provoca obesidad, la cual es un trastorno nutricional que se presenta a cualquier edad (Becerril-Sánchez, 2015, Hernández, 2019).

Por lo que la determinación del GER es necesaria para evaluar este balance y evitar las complicaciones asociadas con la obesidad (Balas Nakash et al., 2008).

En México, la obesidad infantil es un problema de salud pública, la Encuesta Nacional de Salud y Nutrición (ENSANUT) estimó en escolares una prevalencia de sobrepeso y obesidad de $32.8 \%$ para niñas y 33.7\% para niños (Shamah Levy et al., 2018). Dentro de los métodos para evaluar el GER se encuentra la calorimetría indirecta (CI) utilizada en el área clínica y considerada además como un estándar de oro. Sin embargo, este método es costoso y generalmente difícil de realizar si no se cuenta con personal especializado y un espacio adecuado. Es por esto que en la práctica clínica comúnmente se utilizan ecuaciones predictivas para estimar el GER (De la Cruz, De Mateo, 2015; Becerril-Sánchez, 2015).

Existen diversas ecuaciones que han sido propuestas a nivel internacional para la estimación del GER en niños, las cuales han sido validadas contra el GER medido por CI y son específicas para un grupo de edad y diseñadas en ciertos grupos étnicos, sin embargo el uso de las ecuaciones de estimación existentes está limitado por el hecho de que fueron desarrolladas con individuos cuyo ambiente y características físicas difieren a la de la población mexicana, además varían de acuerdo a edad, sexo, raza y composición corporal del grupo estudiado, investigaciones realizadas por Balas-Nakash, concluyeron que el gasto energético en reposo es mayor en escolares con obesidad en comparación de escolares con peso normal (Balas Nakash et al., 2008).

Estos datos concuerdan con otros estudios realizados en niños con y sin obesidad, en donde el GER fue mayor en presencia de obesidad (Rodríguez et al., 2002).

En algunas de las ecuaciones la composición corporal tiene un rol importante sobre el GER ya que se sabe que los niños con obesidad presentan mayor peso corporal, y, por tanto, una cantidad mayor de masa grasa (MG) y masa libre de grasa (MLG) en comparación con niños de peso normal (Vermorel, Lazzer, Bitar et al., 2005). Sin embargo, algunos estudios corroboran lo encontrado en el estudio de Balas-Nakash (2008) acerca de que el GER es igual en niños con peso normal y niños con obesidad cuando se determina la MLG Rodríguez et al., 2002).

Mc Duffie et al., realizaron una comparación de CI con las ecuaciones para GER de Schofield, HarrisBenedict, FAO/OMS/ONU (1985), Tverskaya (1998) y Maffeis (1993) en 502 niños entre 6 y 11 años de raza negra y blanca con normopeso, sobrepeso y obesidad, encontrando que ninguna de las ecuaciones estimó el GER de forma precisa (McDuffie et al., 2004).

En otro estudio Balas-Makash et al., compararon las ecuaciones Schofield, Harris-Benedict, FAO/OMS/ONU y Tverskaya con $\mathrm{CI}$ en 114 escolares mexicanos de 9 a 12 años con y sin sobrepeso y encontrando que la ecuación de mayor concordancia fue la de Tverskaya (Balas Nakash et al., 2008).

Sin embargo, existen otras ecuaciones como la de Lazzer, desarrollada específicamente para niños y adolescentes severamente obesos, la cual ha sido escasamente comparada con las ecuaciones previamente mencionadas (Lazzer, Agosti, et al., 2006).

Por lo que nuestro objetivo es estimar el gasto energético en reposo obtenido por las ecuaciones predictivas de Schofield, Tverskaya y Lazzer comparada con calorimetría indirecta en escolares del estado de Nuevo León de 7 a 12 años con obesidad.

\section{Material y Método}

Se realizó estudio comparativo, descriptivo y trasversal en escolares con obesidad, entre 7 y 12 años de edad, de ambos géneros participantes en un campamento de verano. 
Todos los padres de los escolares participantes firmaron carta de consentimiento informado y carta de asentimiento por parte del niño. Se excluyeron a los que no presentaron diagnóstico de obesidad por medio del IMC según la referencia de la OMS 2007, y a los participantes que presentaran alguna patología crónica (enfermedad del tracto respiratorio y/o cardiopatías) que pudiera alterar los resultados de la determinación, eliminando a los participantes que durante la medición de la calorimetría indirecta presentaron inestabilidad respiratoria $(\mathrm{SO} 2<88 \% \mathrm{o}$ FIO2 > 0.6 o $\mathrm{PaO} 2$ < $60 \mathrm{~mm} \mathrm{Hg}$ o $\mathrm{PaCO} 2$ > $45 \mathrm{~mm}$ $\mathrm{Hg})$.

Procedimiento:

Selección de la muestra.

Muestra no probabilística, invitando a participar en un campamento de verano en las instalaciones del Bioparque Estrella con duración de 7 días las 24 horas del día a escolares de las diferentes escuelas públicas del estado de Nuevo León, a las personas que respondieron a la convocatoria se les realizó una evaluación antropométrica de peso y talla y se seleccionaron para participar en el campamento a todos aquellos que presentaron obesidad de acuerdo al diagnóstico del IMC.

Evaluación antropométrica.

A todos los participantes se les realizó evaluación antropométrica que incluyó peso, masa grasa y masa libre de grasa por medio de bioimpedancia eléctrica a través del inBody770® (InBody CO., LTD, Seoul Korea) y medición de estatura (Estadímetro portátil marca SECA $® 213)$ para obtener el IMC $(\mathrm{kg} / \mathrm{m} 2)$. El diagnóstico nutricional se obtuvo en base a tablas de referencia de la OMS 2007 (obesidad $=+2$ DE).

Evaluación del gasto energético en reposo con calorimetría indirecta

La determinación del GER se realizó por personal entrenado utilizando el calorímetro modelo Quark RMR $®$ (COSMED) el cual fue calibrado al inicio de las mediciones de acuerdo a los requerimientos establecidos por la European Medical Device Directive.

La medición se realizó bajo previo ayuno y reposo absoluto de 12 horas, trasladándose al escolar a la habitación de lectura en un carro de golf para evitar su movilidad. La habitación de lectura permaneció a temperatura constante de $20^{\circ} \mathrm{C}$, la determinación se realizó en una sola ocasión con el niño recostado en posición decúbito supino colocando un casco "Canopy" con capa sobre su cabeza para la recolección de gases por un periodo de entre 25 y 30 minutos.

Durante la medición los niños permanecieron despiertos, sin realizar ningún movimiento que implicara esfuerzo físico, lo más tranquilos posible y respirando por la nariz a través de la mascarilla.

Posteriormente se llevó a cabo la captura de datos para realizar el análisis estadístico.

Evaluación del gasto energético en reposo con ecuaciones de estimación

Las ecuaciones de predicción utilizadas para estimar el GER fueron Schofield, Tverskaya y Lazzer (Tabla 1); las tres ecuaciones se dividen por género. Schofield separa la ecuación en dos grupos de edad, escolares y adolescentes y utiliza los datos de peso en $\mathrm{kg}$ y talla en $\mathrm{cm}$. La ecuación de Tverskaya y Lazzer fueron desarrolladas en población con obesidad y utilizan otros parámetros como es: masa grasa, masa libre de grasa y edad, y determinan el gasto energético en un grupo de edad de 6 a 18 años y de 7 a 18 años respectivamente, La diferencia con la ecuación de Lazzer es que el resultado se obtiene en $\mathrm{KJ}$ y se convirtió a kcal $(1 \mathrm{KJ}=0.24 \mathrm{kcal})$.

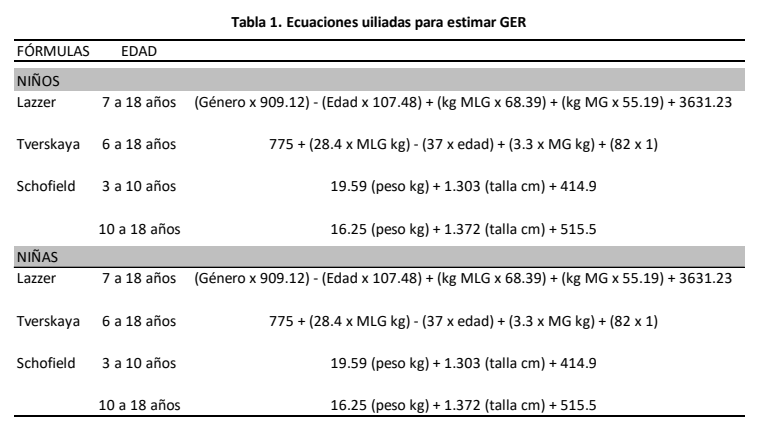

Análisis Estadístico

La creación de la base de datos se realizó con el programa Windows-Excel® 2013 para el análisis estadístico y comparación de las ecuaciones de predicción (Schofield, Tverskaya y Lazzer) versus calorimetría indirecta. El análisis estadístico se realizó son el software MedCalc® versión 13.3, utilizando estadística paramétrica (t student para una 
muestra) para evaluar el error sistemático y el error proporcional (regresión simple) entre las ecuaciones de predicción y la CI.

La equivalencia grupal (diferencias sistemáticas) entre el gasto energético en reposo calculado por las ecuaciones predictivas y la calorimetría indirecta se evaluó con el sesgo o media de las diferencias.

Para comprobar que el sesgo fuera uniforme a lo largo de la magnitud de las mediciones (error proporcional) se realizó un análisis de regresión simple donde la pendiente no fuera significativa (P> $0.05)$.

La equivalencia individual entre el gasto energético calculado con las ecuaciones predictivas y la calorimetría indirecta se determinó mediante un método no paramétrico sugerido por Bland y Altman. Para cada ecuación se calculó el porcentaje de observaciones cuyas diferencias fueran menores a $100,125,150$ y $175 \mathrm{kcal}$. Estos valores en calorías representan los cambios mínimos del gasto energético en reposo para el grupo de edad escolar (Marugán, 2011).

\section{Resultados}

De un total de 43 escolares con obesidad evaluados, se eliminaron 2 sujetos, por lo que se presentan resultados de 41 escolares. De acuerdo al género el $59 \% \quad(\mathrm{n}=24)$ fueron del género femenino $\mathrm{y}$ $41 \%(\mathrm{n}=17)$ del género masculino, con un promedio de edad de $9.0 \pm 2.0$ años. La comparación de cada una de las ecuaciones contra calorimetría indirecta se visualiza en la Figura 1.

En los datos obtenidos de la comparación del GER estimado por Schofield, Tverskaya y Lazzer versus calorimetría indirecta, el promedio de las diferencias fue de $232 \mathrm{kcal}, 89 \mathrm{kcal}$ y $251 \mathrm{kcal}$ respectivamente (Figura 2).

En los límites de concordancia se obtuvieron rangos entre $660 \mathrm{kcal} \mathrm{y}-264 \mathrm{kcal}$ de diferencia, obteniendo en la ecuación de Schofield unos límites entre 660 kcal, -195 kcal, Tverskaya $443 \mathrm{kcal},-264 \mathrm{kcal}$ y Lazzer $624 \mathrm{kcal},-121 \mathrm{kcal}$.

La equivalencia grupal entre el gasto energético en reposo estimado por las ecuaciones predictivas y la calorimetría indirecta se evaluó con el sesgo o media de las diferencias. En resumen, de las tres ecuaciones, todas sobreestiman significativamente el gasto energético en reposo comparado con calorimetría indirecta, esto quiere decir que tuvieron error sistemático, para esto se realizó un análisis de regresión simple donde se obtuvo una significancia para la ecuación predictiva de Schofield $(\mathrm{p}=<0.0001)$, Tverskaya $(\mathrm{p}=<0.0028)$ y Lazzer $(\mathrm{p}=<$ $0.0001)$.

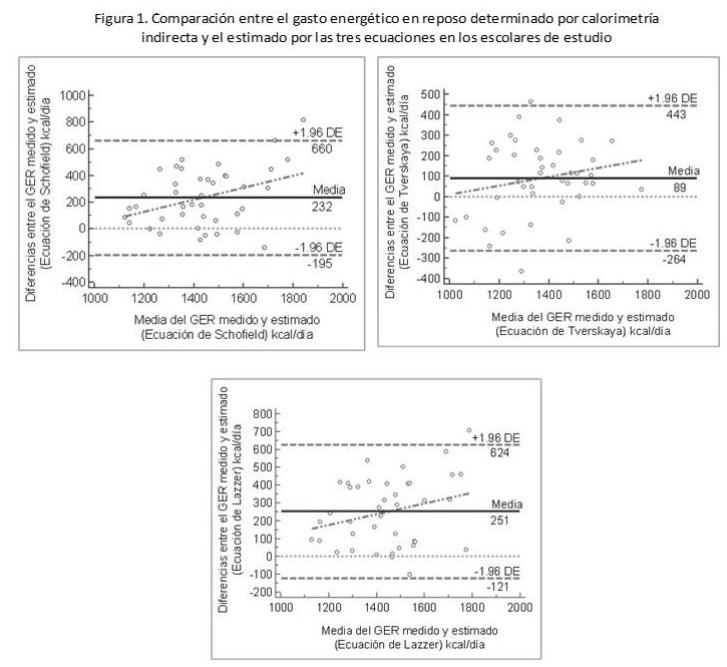

De las tres ecuaciones que se compararon con calorimetría indirecta únicamente la ecuación de Schofield presentó error proporcional $(\mathrm{p}=0.0170)$ lo que quiere decir que las diferencias no fueron homogéneas a lo largo de la magnitud de las mediciones. En cambio, las otras ecuaciones no presentaron error proporcional por lo que no fueron significativas, Tverskaya $(\mathrm{p}=0.2155)$ y Lazzer $(\mathrm{p}=0.0884)$.

La Tabla 2 muestra el promedio de kcal medidas por calorimetría indirecta y estimada para cada una de las ecuaciones de predicción con su desviación estándar del total de la muestra. Todas las ecuaciones sobreestiman el GER de la calorimetría indirecta sistemáticamente. 
Figura 2. Comparación de la media de cada una de las ecuaciones de estimación contra calorimetría indirecta de los sujetos de estudio.

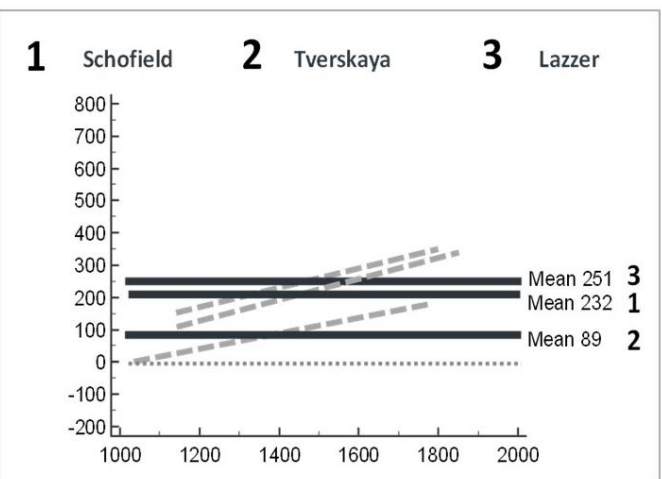

Tabla 2. Comparación del gasto energético estimado mediante ecuaciones de estimación y calorimetría indirecta en los escolares del estudio

\begin{tabular}{lllc}
\hline Método & $\begin{array}{l}\text { Media } \pm \\
\text { DE }\end{array}$ & $\begin{array}{l}\text { Sesgo o error } \\
\text { sistemático }\end{array}$ & $\begin{array}{c}\text { Límites de } \\
\text { concordancia ó } \\
\text { precisión de la } \\
\text { ecuación }\end{array}$ \\
\hline $\begin{array}{l}\text { Calorimetr } 1321 \pm \\
\text { ía indirecta } 172\end{array}$ & - & - & - \\
$\begin{array}{l}\text { Ecuación } 1553 \pm \\
\text { Schofield } 242\end{array}$ & $232^{*}<0.0001$ & $660 /-195$ \\
$\begin{array}{l}\text { Ecuación } 1411 \pm \\
\text { Tverskaya }\end{array} 203$ & $89 *<0.0028$ & $443 /-264$ \\
$\begin{array}{l}\text { Ecuación } 1572 \pm \\
\text { Lazzer }\end{array}$ & 217 & $251^{*}<0.0001$ & $624 /-121$ \\
\hline
\end{tabular}

Por otro lado, se establecieron los límites del porcentaje acumulado (Tabla 3 ) de las diferencias en cada uno de los rangos, en base a las calorías que representan los cambios mínimos del gasto energético en reposo por cada grupo de edad.

Tabla 3. Porcentaje acumulado de diferencias entre el gasto energético por las ecuaciones de predicción versus por calorimetría indirecta

Porcentaje acumulado de diferencias

\begin{tabular}{lcccccc}
\hline Ecuación & & $<100 \mathrm{kcal}$ & $<125 \mathrm{kcal}$ & $<150 \mathrm{kcal}$ & $<175 \mathrm{kcal}$ & $<300 \mathrm{kcal}$ \\
\cline { 3 - 7 } Schofield & 29 & 34 & 39 & 46 & 58 \\
Tverskaya & 29 & 44 & 49 & 54 & 90 \\
Lazzer & 29 & 32 & 37 & 39 & 56 \\
\hline
\end{tabular}

De acuerdo a los datos, se observa que en 1 de cada 3 sujetos las ecuaciones estiman el gasto energético en reposo comparado con $\mathrm{CI}$ con un rango de exactitud por debajo de $100 \mathrm{kcal}$ de diferencia, en los valores siguientes para cada uno de los límites por debajo de $125 \mathrm{kcal}, 150 \mathrm{kcal}$ y $175 \mathrm{kcal}$ la ecuación que más porcentaje acumulado de casos tiene es la de Tverskaya. Incluso en esta ecuación el $90 \%$ de los casos cae por debajo de las $300 \mathrm{kcal}$ de diferencia con la CI, comparado con las ecuaciones de Schofield y Lazzer donde apenas el $50 \%$ de los casos presenta diferencias en el gasto energético en reposo por debajo de las 300 kcal de diferencia comparado con la calorimetría indirecta.

\section{Discusión}

En el presente estudio decidimos evaluar el gasto energético en reposo estimado por las ecuaciones de predicción (Schofield, Tverskaya y Lazzer) versus calorimetría indirecta en escolares con obesidad, donde la hipótesis planteada fue que ninguna de las ecuaciones utilizadas es precisas ni exactas comparadas con la calorimetría indirecta en escolares mexicanos de 7 a 12 años de edad con obesidad. Esto se justifica porque las ecuaciones que existen y se utilizan actualmente en nuestra población para estimar el gasto energético en reposo fueron desarrolladas con individuos residentes de países industrializados, cuyo ambiente y características físicas difieren a la de la población mexicana, además varían de acuerdo a la edad, sexo, raza y composición corporal del grupo estudiado (Balas Nakash et al., 2008).

Los resultados de este estudio muestran que a pesar de que las tres ecuaciones sobreestiman significativamente el gasto energético en reposo comparado con calorimetría indirecta, en términos grupales la ecuación de Tverskaya es la más recomendable por ser la que muestra una media de diferencia menor de $89 \mathrm{kcal}$ respecto a los datos obtenidos por calorimetría indirecta. Lo anterior similar al estudio publicado por Balas-Nakash et al., (2008) donde también encontraron que la ecuación de Tverskaya es de las que más se aproxima al GER. En la ecuación de Schofield el promedio de las diferencias fue de $232 \mathrm{kcal}$, por lo que sobreestima el gasto energético en reposo comparado con la calorimetría indirecta. En los límites de concordancia tiene un límite máximo de 660 calorías y un límite mínimo de -195 calorías de diferencia con la 
calorimetría, lo que nos dice que el $95 \%$ de los casos puede caer dentro de estos límites. La línea de regresión o pendiente, presentó error proporcional lo cual muestra que no se distribuyó de manera homogénea a lo largo de la magnitud de las mediciones, esto se puede justificar ya que es una ecuación que se desarrolló en población con normopeso, no en niños con obesidad. Nuestro resultado concuerda con el publicado por Derumeaux-Burel en el 2004 donde también encontraron que la ecuación de Schofield sobreestimaba el gasto energético real.

En la ecuación de Tverskaya se observa que el sesgo es menor que la ecuación de Schofield respecto a la calorimetría indirecta. De igual manera sobreestima el gasto energético en reposo y los límites de concordancia son más estrechos. Las diferencias se distribuyeron de manera homogénea a lo largo de la magnitud de las mediciones por lo que estadísticamente no hay error proporcional.

Dentro de los resultados con la ecuación de Lazzer, se obtuvo una media en las diferencias de la estimación del gasto energético en reposo más alta que en las ecuaciones de Schofield y Tverskaya, con promedio de $251 \mathrm{kcal}$ y límites de concordancia más amplios que oscilan entre 624 y $-121 \mathrm{kcal}$ lo cual indica que la ecuación no es muy precisa comparado con la calorimetría indirecta. La pendiente o línea de regresión no mostró error proporcional.

A diferencia de nuestro resultado, en el estudio publicado en el 2006 por Lazzer, et al., para niños y adolescentes severamente obesos, la ecuación que desarrollaron, la cual fue comparada el siguiente año por el mismo autor, con las ecuaciones de McDuffie, Derumeaux, Tverskaya, Schofield, FAO/WHO/UNU y Harris-Benedict, resultó ser la de mayor aproximación (Lazzer, Agosti, et al., 2007).

Así mismo, Marra M et al (2015) determinó que la ecuación que mejor estima el GER en adolescentes de 14 a 18 años es la de Lazzer. Sin embargo, en estos estudios fueron incluidos niños $\mathrm{y}$ adolescentes obesos de 12 a 18 años, rango de edad que no se incluye en el presente estudio, por lo que podría ser que para adolescentes Lazzer sea la más precisa.

Aunque la ecuación de Tverskaya es la que menores diferencias grupales en promedio mostró respecto a la calorimetría indirecta, esta ecuación podría recomendarse para estudios poblacionales en niños con obesidad ya que su exactitud es buena a nivel grupal, en forma individual o en la práctica clínica se tendría que revisar si la ecuación ofrece estimaciones confiables para realizar una intervención, y aun así sería cuestionable dado los límites de concordancia, ya que entre más estrecho sea el rango más precisa es la ecuación.

Así mismo pudimos corroborar que todas las ecuaciones comparadas con calorimetría indirecta sobreestiman el gasto energético, por lo que, de contar con ella, la mejor herramienta para determinar el GER en la población infantil con obesidad es la calorimetría indirecta.

Sin embargo, es necesario seguir realizando más estudios, donde se incluyan todas las ecuaciones existentes y que además las poblaciones sean estadísticamente representativas. Ya que para que se logre una mejora en el estado nutricional de los escolares es necesario que se establezcan acertadamente sus requerimientos o gasto de energía, para no sobre o subestimar sus necesidades y con ello asegurar que su nutrición y crecimiento sean los adecuados.

\section{Conclusiones}

Se concluye que para determinar el GER en la población infantil con obesidad la mejor herramienta es la calorimetría indirecta. En base a los resultados obtenidos, en la evaluación grupal se concluye que las tres ecuaciones de predicción sobreestiman significativamente el gasto energético en reposo comparado con calorimetría indirecta.

En la evaluación individual, se concluye que los límites de concordancia mostraron variaciones al compararla con la calorimetría.

La ecuación de Tverskaya fue la que presentó menor error sistemático comparado con calorimetría indirecta, sin embargo, esta se recomienda únicamente a nivel poblacional ya que su exactitud es buena a nivel grupal pero no a nivel individual. 


\section{Bibliografía}

Balas-Nakash M., Villanueva-Quintana a., VadilloOrtega F., et al. (2008). Validación del uso de fórmulas para estimar el gasto energético en reposo en niños mexicanos de 9 a 12 años de edad con y sin obesidad. Revista de Investigación Clínica. 60(5), 395-402. https://biblat.unam.mx/en/revista/revista-deinvestigacion-clinica

Becerril-Sánchez ME, Flores-Reyes M, RamosIbáñez N, Ortiz-Hernández L. (2015) Ecuaciones de predicción del gasto de energía en reposo en escolares de la Ciudad de México. Acta Pediatr Mex; 36, 147-157. http://www.scielo.org.mx/scielo.php?script=sci_ arttext\&pid=S0186-23912015000300003

De la Cruz MS, de Mateo Silleras B, Alicia Camina Martin M, Enciso L, de la Torre A, Paz Redondo del Rio M, et al., (2015). Concordancia entre calorimetría indirecta y modelos predictivos en una población sana española. Nutr Hosp. 32(2): 888-896. DOI: 10.3305/nh.2015.32.2.9162

Derumeaux-Burel Hélène, Meyer Martine, Morin Liliane., et al. (2004). Prediction of resting energy expenditure in a large population of obese children. Am J Clin Nutr; 80:1544-50. DOI: 10.1093/ajcn/80.6.1544

Hernández-Ortega A, Osuna Padilla IA, RendónRodríguez R, Narváez-Velázquez PB, ChávezGonzález MJ, Estrada-Velasco BI. (2019) Exactitud de las ecuaciones predictivas del gasto energético basal: estudio transversal en niños y adolescentes con sobrepeso y obesidad de Morelos, México. Rev Esp Nutr Hum Diet. 23(2). DOI: 10.14306/renhyd.23.2.706.

Lazzer Stefano, Agosti Fiorenza, De Col Alessandra, et al., (2006) Development and cross-validation of prediction equations for estimating resting energy expenditure in severely obese Caucasian children and adolescents. British Journal of Nutrition; 96; 973-979. DOI: 10.1017/BJN20061941

Lazzer S, Agosti F, De Col A., et al. (2007). Comparison of predictive equations for resting energy expenditure in severely obese Caucasian children and adolescents. J Endocrinol Invest.; 30(4): 313-7. DOI: 10.1007/BF03346298

Marra Maurizio, Montagnese Concetta, Sammarco Rosa., et al. (2015). Accuracy of Predictive Equations for Estimating Resting Energy Expenditure in Obese Adolescents. The Journal of pediatrics, J Pediatr; 166:1390-6. DOI: 10.1016/j.jpeds.2015.03.013

Marugán de Miguelsanz J.M., Redondo Del Río P., De Mateo Silleras B. (2011). Balance energético en el niño. Pediatr Integral; XV (4):369-373. https://www.pediatriaintegral.es/wpcontent/uploads/2012/03/Pediatria-Integral-XV4.pdf.

McDuffie JR., Adler-Wailes D., Elberg J., et al. (2004). Prediction equations for resting energy expenditure in overweight and normal-weight black and White children. Am J Clin Nutr; 80: 365-73. DOI: 10.1093/ajcn/80.2.365.

Rodríguez G, Moreno LA, Sarria A, Pineda I, Fleta J, Pérez-González JM, et al. (2002). Determinants of resting energy expenditure in obese and non-obese children and adolescents. $J$ Physiol Biochem, 58(1): 9-15.

Shamah T., (2018). Sobrepeso y obesidad en niños y adolescentes en México, actualización de la Encuesta Nacional de Salud y Nutrición de Medio Camino 2016. Salud Pública Mex, 60(3):244-256. DOI: $10.21149 / 8815$

Schofield WN. (1985). Predicting basal metabolic rate, new standards and review of previous work. Hum Nutr Clin Nutr. 39; Suppl 1:5-41.

Stubelj M, Teraž K, Poklar Vatovec T. (2020). Predicting equations and resting energy expenditure changes in overweight adults. $Z d r$ Varst.; 59(1):33-41. DOI: 10.2478/sjph-20200005

Tverskaya R, Rising R, Brown D, et al. (1998). Comparison of several equations and derivation of a new equation for calculating basal metabolic rate in obese children. J Am Coll Nutr. 17(4):3336 
Calorimetría indirecta, Ecuaciones predictivas,

Pediatría, Obesidad, Gasto energético en reposo.

Vermorel M, Lazzer S, Bitar A, Ribeyre J, Montaurier C, Fellamn N, et al. (2005) Contributing factors and variability of energy expenditure in nonobese, obese and postobese adolescents. Reprod Nutr Dev; 45(2): 129-42.

DOI: $10.1051 / \mathrm{rnd}: 2005014$ 